\title{
Analysis of nanostructured porous films by measurement of adsorption isotherms with optical fiber and ellipsometry
}

\author{
Alberto Álvarez-Herrero, Hector Guerrero, Eusebio Bernabeu, and David Levy
}

\begin{abstract}
An optical method to determine the nanostructure and the morphology of porous thin films is presented. This procedure is based on the response of a side-polished optical fiber with the film under study, when an adsorption-desorption cycle is carried out. Spectroscopic ellipsometry provides additional information about the optical properties and adsorption behavior of the film. Pore size distribution, anisotropy, and inhomogeneity of films can be determined by use of these two complementary techniques. To check the performances and suitability of the optical method, we have characterized a typical porous material: a $\mathrm{TiO}_{2}$ film deposited by evaporation. Water vapor has been used for the adsorption cycles. The well-known columnar structure of the evaporated $\mathrm{TiO}_{2}$ has been evidenced, and the relation between the nanostructure and the optical properties of the film is showed. (C) 2002 Optical Society of America

OCIS codes: $310.6860,060.2370,120.2130$.
\end{abstract}

\section{Introduction}

The nanostructure of materials determines their optical properties and behavior (i.e., refractive index and stability under different environment conditions). This fact has great importance in the design and manufacturing process of optical coatings. To predict the response of optical devices such as inteferencial filters or antireflection coatings, it is necessary to know their nanostructure and the repercussion in their optical features. In addition, knowledge of the relations between the optical properties and the nanostructure of films is useful to develop optical sensors.

The nanostructure of thin films (also called microstructure in the literature) deposited by evaporation techniques has been extensively described, and many studies have been carried out to understand the

\footnotetext{
A. Álvarez-Herrero (alvareza@inta.es), H. Guerrero, and D. Levy are with the Laboratorio de Instrumentación Espacial, Instituto Nacional de Técnica Aeroespacial, 28850 Torrejón de Ardoz, Madrid, Spain. D. Levy is also with the Instituto de Ciencia de Materiales de Madrid, Consejo Superior de Investigaciones Científicas, 28049 Cantoblanco, Madrid, Spain. E. Bernabeu is with the Departamento de Óptica, Facultad de Ciencias Físicas, Universidad Complutense de Madrid, 28040 Madrid, Spain.

Received 31 January 2002; revised manuscript received 10 June 2002.

0003-6935/02/316692-10\$15.00/0

(C) 2002 Optical Society of America
}

growth process and the influence of the deposition conditions on the properties of the coatings. ${ }^{1,2}$ Cross sections of micrographs from scanning electron microscopy and transmission electron microscopy have provided direct information about the nanostructure of the multilayer systems, although many other techniques have been utilized to characterize films (x-ray diffraction, electron diffraction, Auger electron spectroscopy, x-ray fluorescence, etc.). It is currently known that most evaporated films are formed by a nanostructure composed of nanometric-size columns, amorphous or crystalline. The compactness ratio and the direction and size of columns are determined by conditions present during the evaporation process.

The temperature of the substrate is a determining parameter of the nanostructure of the film. Movchan and Demchishin ${ }^{3}$ observed that the morphology of the structure was connected to the ratio of the substrate temperature to the melting point of the deposited material: the normalized temperature $T_{s} / T_{m}$. Three zones were distinguished with different structure properties depending on normalized temperature. Zone $1 \mathrm{films}\left(T_{s} / T_{m}<0.3\right)$ show a columnar morphology with domed tops that are separated by voided boundaries. Zone 2 films $(0.3<$ $\left.T_{s} / T_{m}<0.5\right)$ consist of columnar grains separated by distinct, dense, intercrystalline boundaries and low superficial roughness. Zone 3 films $\left(0.5<T_{s} / T_{m}<\right.$ 1) consist of equiaxed grains with a bright surface. This structure zone model (SZM) was revised by 
Thornton 4 to include sputtering and gas pressure as parameters. Messier et al. ${ }^{5}$ found that the physical structure evolves when the thickness is increased. The columnar structures usually have a conical shape, and they result from a competitive growthdeath process. Therefore the SZM has three parameters: substrate temperature, bombing gas pressure, and thickness. All of them are linked to the mobility of atoms that are added to the structure. Ballistic aggregation theories are a powerful method for understanding the common growth mechanism in the preparation of evaporated films. ${ }^{6,7}$

The well-known columnar structure of evaporated films is a clear example of optical anisotropy and inhomogeneity induced by a typical nanostructure. Films evaporated at normal incidence are expected to have uniaxial birefringence with the optical axis parallel to the columns' direction. These anisotropic coatings have been studied, and interesting theoretical and empirical results have been obtained. ${ }^{8-10}$ The characterization of the inhomogeneity of the optical constants of films that is produced by the variation of the packing density with the thickness has also been considered in many studies. ${ }^{11-14}$ The columnar nanostructure is a porous structure in which voids appear between clusters of molecules and between columns. Through the introduction of theoretical models of the multilayer systems and taking into account a dependency of the refractive index on the layers' thicknesses, it is possible to extract accurate information about the optical properties of thin films.

Another consequence of the films' porosity is the influence of relative humidity $(\mathrm{RH})$ on the optical properties. The effective refractive index of materials will change with water adsorption in the porous nanostructure. ${ }^{15}$ Hence the change of the optical constants will depend on the size and the shape of the pores. ${ }^{16}$ It is necessary to have nondestructive inspection methods to determine optical constants of the film and to predict their dependence on the film's nanostructure.

In this paper a method to characterize the nanostructure of a film is presented. A novel, to our knowledge, procedure for determining the pore size distribution of the layer by use of side-polished optical fiber (SPF) ${ }^{17}$ is introduced; the procedure is based on adsorption isotherms. ${ }^{18}$ A complete optical analysis of the film is carried out when the results obtained from the SPF device and from ellipsometric measurements are contrasted. The analysis permits the establishment of a relation between the film's nanostructure and its optical behavior. For transparent materials, spectroscopic ellipsometry is sensitive to changes in the refractive index that occur along the thickness of the film. Hence this technique provides information about the global properties of the film: pore size distribution, refractionindex profile, and roughness. ${ }^{16,19-22}$ In addition, a SPF device with the film under study acting as overlayer provides information about local properties of the film in the area that is closest to the fiber core: local pore size distribution and local optical properties. This is due to the high sensitivity of these devices to refractive-index changes in the fiber-film interface.

Because $\mathrm{TiO}_{2}$ is a high-refractive-index material widely used in optical coatings, a film of evaporated titania has been chosen to perform a verification of the nanostructure determination procedure.

\section{Theory}

\section{A. Side-Polished Fiber Devices}

These systems are composed of two elements: an optical fiber whose cladding has, partially or completely, been removed and a film deposited over the $\mathrm{SPF}$. The transmission response of these devices is determined by the evanescent coupling of the light from the fiber to the film. The fiber's guided mode is evanescent in the film (decreasing exponential function), and it makes the device sensitive to the properties of the film only in the interaction area in contact with the fiber core. When the effective index of the fiber's guided mode is equal to the effective index of the highest-order mode of the waveguide overlay, the phase-matching condition is satisfied. The spectrum wavelengths of the light transmitted by the optical fiber, which satisfy this phasematching condition, will be coupled to the waveguide overlayer, and therefore they will become attenuated in the transmission signal. These wavelengths are called resonances. Owing to the dependence of the effective index of the film's guided modes on the thickness and the refractive index of the layer, a change in these parameters results in a shift of the resonance wavelength.

The eigenvalue equation for the $m$ th mode propagating in the direction of the fiber axis for an asymmetrically planar waveguide is ${ }^{23}$

$$
\frac{2 \pi d}{\lambda}\left(n_{\mathrm{o}}{ }^{2}-n_{\mathrm{eo}}{ }^{2}\right)^{1 / 2}=m \pi+\phi_{1}+\phi_{2},
$$

where $n_{\mathrm{o}}$ is the refractive index of the overlay material of the waveguide overlay, $n_{\mathrm{eo}}$ is the effective index of the propagating mode, $\lambda$ is the input wavelength, $d$ is the overlayer thickness, and $m$ is an integer. $\phi_{1}$ and $\phi_{2}$ are given by

$$
\phi_{i}=\tan ^{-1} \mathrm{~s} \frac{\left(n_{\mathrm{eo}}{ }^{2}-n_{i}{ }^{2}\right)^{1 / 2}}{\left(n_{\mathrm{o}}{ }^{2}-n_{\mathrm{eo}}{ }^{2}\right)^{1 / 2}}, \quad i=1,2,
$$

where $n_{1}$ is the fiber cladding's refractive index, $n_{2}$ is the external refractive index, and $\zeta$ is equal to 1 for TE polarization and $\left(n_{\mathrm{o}} / n_{i}\right)^{2}$ for TM polarization. Assuming that the film is anisotropic, uniaxial with the optical axis normal to the surface (column directions), different values of the refractive index of the layer for TE and TM polarizations have to be considered $\left(n_{\mathrm{OTM}}, n_{\mathrm{oTE}}\right)$. The index-matching condition is satisfied when $n_{e o}=n_{e f}$, where $n_{e f}$ is the effective index of the propagating mode of the optical fiber.

Hence the expressions for TM and TE resonances 
obtained from consideration of the index-matching condition are

$$
\begin{gathered}
\lambda_{\mathrm{TM}}=\frac{2 \pi d \sqrt{n_{\mathrm{oTM}}{ }^{2}-n_{\mathrm{ef}}{ }^{2}}}{m \pi+\phi_{1}{ }^{\mathrm{TM}}+\phi_{2}^{\mathrm{TM}}}, \\
\lambda_{\mathrm{TE}}=\frac{2 \pi d \sqrt{{n_{\mathrm{oTE}}}^{2}-n_{\mathrm{ef}}{ }^{2}}}{m \pi+{\phi_{1}}^{\mathrm{TE}}+\phi_{2}{ }^{\mathrm{TE}}},
\end{gathered}
$$

where the refractive index of the layer for TE or TM polarization has been introduced in the Eqs. (1) and (2) depending on the considered mode. It is necessary to highlight the importance of taking into account the dispersion law of the refractive indices of all the components of the system (core fiber, cladding fiber, and film) in the calculation of the resonances along the spectrum.

In the case of our SPF device, the overlayer is porous. For porous materials, adsorption of water molecules and capillary condensation occur when the $\mathrm{RH}$ of the environment increases. The process of filling the pores with water takes place in three steps. First, water-vapor molecules are physically adsorbed on the walls of the pores owing to the action of shortrange interaction forces between adsorbent and adsorbate. In the second step, when the $\mathrm{RH}$ increases, capillary condensation of water in the pores takes place. The last step starts when the pores are completely filled with water, and bulk condensation begins over the external surface of the solid.

The water-filling process of the pores causes an increase in the effective refractive index of the porous material. The adsorbent-adsorbate system can be described as a material-air-water mixture. The volume fraction of water increases during the filling, and the volume fraction of air decreases, resulting in a higher value of the effective refractive index. The refractive-index variation consequently causes a shift in the wavelength of the resonance. This shift is approximately proportional to the variation of the refractive index of the layer for small variations. This assumption was checked by use of Eq. (7) to calculate the resonance shift of the sixth TE mode versus the overlayer's refractive index. The calculation was carried out by consideration of $n_{\mathrm{ef}}=1.46$ and $d=1212 \mathrm{~nm}$. All these values are similar to the $\mathrm{TiO}_{2}-\mathrm{SPF}$ system under study. The result of the simulation is showed in Fig. 1. The linear relation of the refractive index of the layer and the resonance shift for changes of the refractive index as high as 0.1 can be observed. So it can be concluded that the shift of the resonances is proportional to the refractive index and therefore to the amount of water adsorbed by the overlayer. ${ }^{16}$

The curves of the resonance shift against the $\mathrm{RH}$ at a controlled temperature are adsorption isotherms, and they are determined by the shape and the size of the pores. Hence the nanostructure of the material can be derived by measurement of the response of optical properties to changes of $\mathrm{RH}$. This fact will be

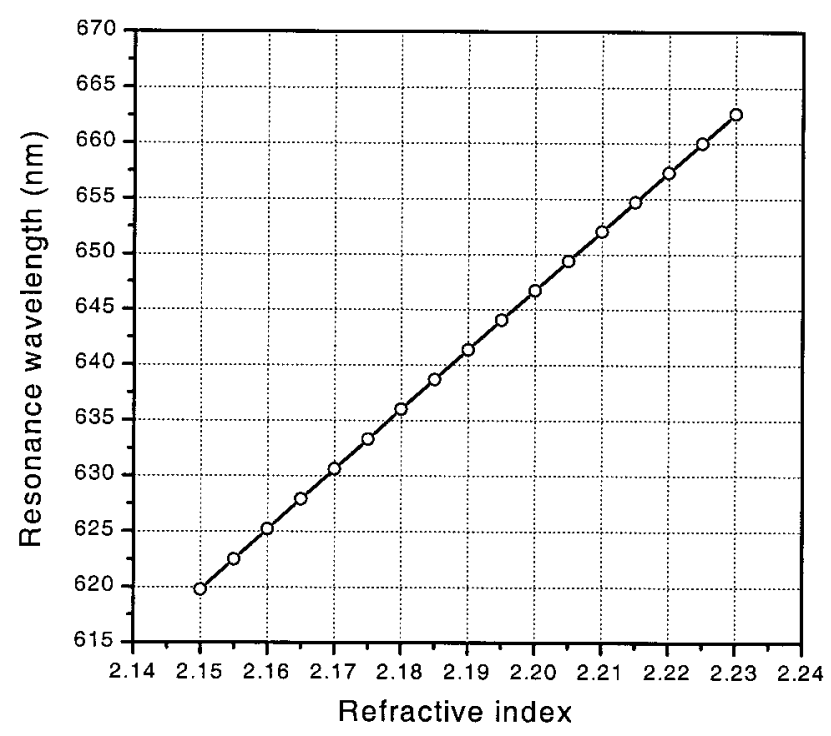

Fig. 1. Simulation of the $\mathrm{TiO}_{2}-\mathrm{SPF}$ system. Resonance shift of the sixth TE mode versus the overlayer's refractive index by use of Eq. (7) and consideration of $n_{\mathrm{ef}}=1.46$ and $d=1212 \mathrm{~nm}$. The linear relation of the refractive index of the layer and the resonance shift for changes of the refractive index as high as 0.1 can be observed.

used in Subsection 2.C to calculate the pore size distribution of the material under study.

\section{B. Ellipsometric Measurements of Water Adsorption}

It is known that the light flux transmitted by an ideal rotating-polarizer ellipsometer is ${ }^{24}$

$$
I(t)=I_{0}\left(1+\alpha \cos 2 \omega_{p} t+\beta \sin 2 \omega_{p} t\right),
$$

where $\omega_{p}$ is the rotation frequency of the polarizer.

A Fourier or Hadamard transform is usually used to determine $\alpha$ and $\beta$. The ellipsometric parameters $\Psi$ and $\Delta$ can be extracted from these magnitudes because they verify the following expressions:

$$
\begin{aligned}
\tan \Psi & =\left(\frac{1+\alpha}{1-\alpha}\right)^{1 / 2} \tan A, \\
\cos \Delta & =\frac{\beta}{\left(1-\alpha^{2}\right)^{1 / 2}},
\end{aligned}
$$

where $A$ is the analyzer angle and the ellipsometer parameters are defined as

$$
\tan \Psi \exp (i \Delta)=\frac{r_{\|}}{r_{\perp}},
$$

where $r_{\|}$and $r_{\perp}$ are, respectively, the Fresnel reflection coefficients parallel and perpendicular to the incident plane.

A change in the $\mathrm{RH}$ of the environment produces variations in the amount of water adsorbed in the pores of the film, as was explained in Subsection 2.A. It also produces a change in the ellipsometric parameters, $\Psi$ and $\Delta$; therefore information about the water adsorbed in the pores of the material can be ex- 
tracted. Linear regressions with theoretical models that consider Bruggemann models of the effective refractive index permit us to obtain the profile of water penetration in the material and to detect the bulk condensation over the surface. ${ }^{15}$ Besides, the magnitudes $\tan \Psi$ and $\cos \Delta$ have a linear relation with the refractive index of the material for variations as great as $\delta n \leq 0.04$ and therefore with the amount of water adsorbed. ${ }^{16}$ Hence the curves of variations of the ellipsometric parameters against the $\mathrm{RH}$ at a controlled temperature are adsorption isotherms. This important conclusion will be utilized to extract the pore size distribution of the film.

\section{Pore Size Distribution}

As has been explained in Subsections 2.A and 2.B, two measurable optical magnitudes, the shift of resonances and the ellipsometric parameters, are proportional to the amount of water adsorbed in the porous material. It permits us to utilize the Pierce $\operatorname{method}^{18}$ to calculate the pore size distribution from data obtained from the film behavior at various environments of $\mathrm{RH}$ and equal thermal conditions; that is, from the adsorption isotherms. As far as we know, the application of the Pierce method to the shift of the resonance of a SPF device is an original contribution. This method is based on the Kelvin equation

$$
\ln \mathrm{RH}=-\frac{2 \gamma V_{L}}{R T} \frac{1}{r_{m}},
$$

where $\gamma$ is the water surface tension, $V_{L}$ is the molar volume, $R$ is the perfect gases' constant, $T$ is the temperature, and $r_{m}$ is the radius of the pore minus the thickness of adsorbed film on the walls. This expression establishes a relation between the $\mathrm{RH}$ and the pore radius in which the capillary condensation occurs. There is an extensive description of the Pierce method in Ref. 18, and we have applied this method to ellipsometry in Vycor glasses. ${ }^{16}$

We perform water adsorption cycles because we are interested in the behavior of the materials under changes of $\mathrm{RH}$ at room conditions. The water adsorption cycles provide information about the stability of optical components and the possibility of developing RH optical sensors. Nevertheless, in the literature it is generally assumed that water vapor is not the most suitable gas for pore size determination. Water is a polar molecule with strong intermolecular forces. This fact facilitates the growth of multilayers of adsorbed molecules in the pores during the adsorption process, even though the monolayer is still incomplete in other places of the surface. Therefore the results obtained can be considered only as an estimation of the pore size. Besides, it is necessary to take into account that

1. Water adsorption isotherms were carried out under normal conditions and therefore without an outgassing process. The impurities adsorbed in the pores had not been removed, and the apparent radius pore is smaller than the real one.

2 . The procedure described in Ref. 16 to correct the adsorption on the pores' walls before capillary condensation takes place is an approximation.

\section{Experiment}

\section{A. Sample Preparation}

\section{1. $\mathrm{TiO}_{2}$ Film Deposition}

The analysis was performed for a $\mathrm{TiO}_{2}$ layer because it is a standard high-refractive-index material used in optical coatings. An evaporation method was selected to deposit the film because this technique usually produces columnar structures. The material was deposited on a glass substrate (sample 1) and on a SPF (sample 2) by electron-beam reactive evaporation in a Leybold A700Q evaporation plant. Both samples were deposited simultaneously in the same evaporation session. The starting material was a TiO tablet of $1 \mathrm{~g}$ provided by Merck. It was melted by use of an electron-beam gun and evaporated for oxygen partial pressure of $2 \times 10^{-4} \mathrm{mbar}$, and the ultimate vacuum obtained was $2 \times 10^{-6} \mathrm{mbar}$. The substrate temperature $\left(200^{\circ} \mathrm{C}\right)$ during the deposition was less than the usual temperature $\left(260^{\circ} \mathrm{C}\right)$ in order to obtain a stronger columnar structure and therefore to engage the effects in the optical properties. The normalized temperature was $T_{s} / T_{m}=0.109$, and it corresponds to that in zone 1 in the SZM, which predicts a columnar morphology with domed tops. The optical thickness of the evaporated layers was measured with photometric techniques, resulting in $20 \lambda / 4$ at $450 \mathrm{~nm}$.

\section{Side-Polished Fiber Probe}

The optical fiber employed was single mode with a cutoff wavelength at $540 \mathrm{~nm}$ (Fiber Core, SM 600). The thickness of the cladding remaining between the dielectric layer and the core of the fiber was approximately $1.00 \pm 0.25 \mu \mathrm{m}$. The interaction length between the optical fiber and the film was measured to be approximately $14.8 \pm 0.1 \mathrm{~mm}$. To polish the optical fiber properly, we placed it inside a V-groove cavity (radius $\approx 425 \pm 25 \mathrm{~mm}$ ) in a quartz substrate and glued it with an epoxy resin. This component was acquired from the Departamento de Física Aplicada (Universidad de Zaragoza, Spain). The effective index of the fiber was obtained prior to the deposition of the overlayer when different Cargille refractive-index liquids were placed over the SPF, and the maximum attenuation was detected. The value obtained was $n_{D}=1.460 \pm 0.004$.

\section{B. Experimental Setup}

\section{Spectrometric Ellipsometry}

A rotating-polarizer spectroscopic ellipsometer (SOPRA, ES-4G) was used to measure the thickness and the optical constants of sample 1. The ellipsometer's nominal repeatability was 0.005 for the ellipsometric 


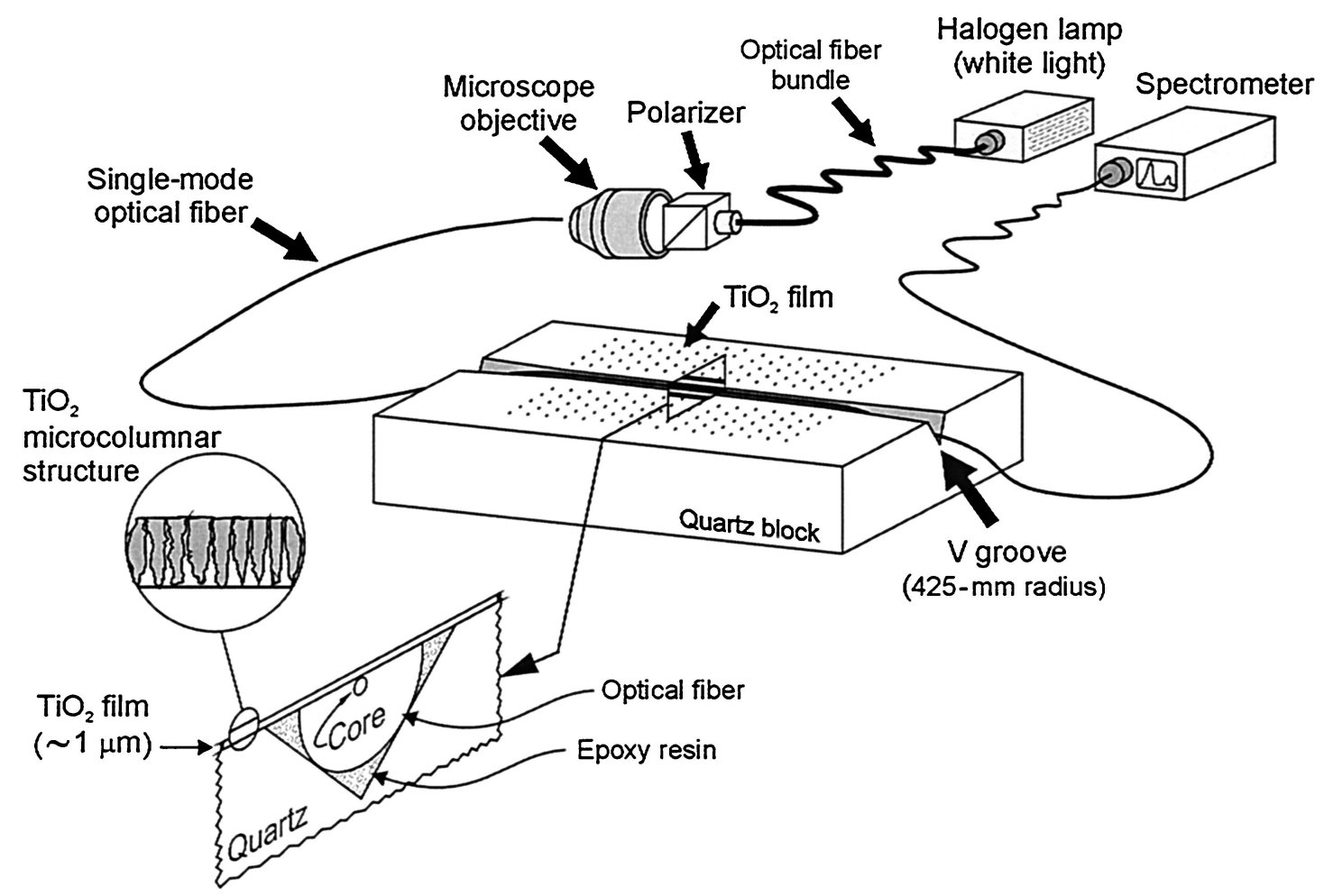

Fig. 2. Experimental setup for measuring the resonance shift of the coupling modes between the SPF and the dielectric film.

parameters $\tan \Psi$ and $\cos \Delta$, and the spectral range employed was $400-800 \mathrm{~nm}$.

The sample was measured at three incidence angles $\left(54.93^{\circ}, 64.95^{\circ}\right.$, and $\left.69.95^{\circ}\right)$ to determine the possible anisotropy and inhomogeneity of the layer. The $\mathrm{RH}$ and the temperature during the measurement were $30 \% \pm 2 \%$ and $23{ }^{\circ} \mathrm{C} \pm 1{ }^{\circ} \mathrm{C}$, respectively.

A climatic chamber, specially designed to be coupled to the ellipsometer at a $70^{\circ}$ incidence angle, was used to perform in situ measurements of the change of optical properties with RH. The stability of the chamber is $\pm 1 \%$ for $\mathrm{RH}$, and its working range goes from $7 \%$ to $90 \% \mathrm{RH}$. The temperature during the measurement was $25.5^{\circ} \mathrm{C} \pm 0.5^{\circ} \mathrm{C}$. Temperature and $\mathrm{RH}$ inside the chamber were measured with a commercial sensor (VAISALA, PHM 233 with the probe $\mathrm{HMP} 46$; accuracy of $0.2{ }^{\circ} \mathrm{C}$ and $1 \% \mathrm{RH}$ ).

\section{Coupling Modes in the Side-Polished Fiber Device}

The experimental setup for measuring the resonances of the coupling modes between the fiber and the dielectric film is showed in Fig. 2. A white-light beam emitted by a halogen lamp is injected into the single-mode optical fiber by use of a microscope objective and is detected with a spectrometer (Instrument Systems, Model Spectro 320). A Glan-Thompson polarizer was placed between the microscope objective and the optical fiber to permit us to select TE or TM mode resonances. An environmental chamber was used to change the humidity conditions of the SPF device. The temperature and $\mathrm{RH}$ were measured with a standard commercial sensor (HMI 31 from VAISALA; ac- curacy of $0.3{ }^{\circ} \mathrm{C}$ and $2 \%$ in temperature and $\mathrm{RH}$, respectively).

\section{Results and Discussion}

\section{A. Optical Properties}

\section{Spectroscopic Ellipsometry}

To extract information about the optical properties and the thickness of the film from the ellipsometric measurements, we propose the following theoretical model (Fig. 3). The $\mathrm{TiO}_{2}$ film is considered inhomogeneous, with superficial roughness. Inhomogeneity is simulated by a 20 -sublayer model with a linear gradient of refractive index that is calculated by the introduction of a variable concentration of void de-

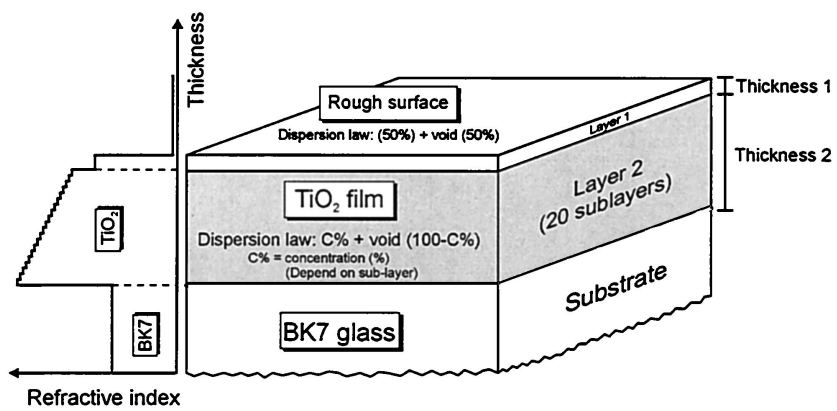

Fig. 3. Ellipsometric model of the film. The $\mathrm{TiO}_{2}$ layer was considered inhomogeneous, with superficial roughness. The inhomogeneity was simulated by use of a variable concentration of voids (100-C\%) along the thickness of the layer. 
Table 1. Regression Results for the Inhomogeneous Model

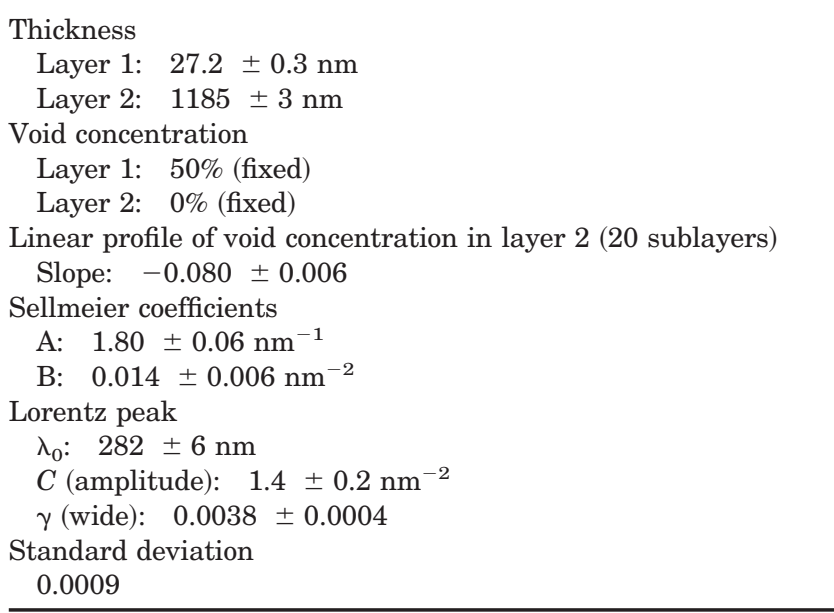

pending on the layer number. The refractive index of every layer was calculated with the Bruggeman model of effective index and the Sellmeier dispersion law with a Lorentz absorption peak at $282 \mathrm{~nm}$. Hence the dispersion law expression was the following:

$$
\begin{aligned}
& \epsilon_{r}=1+\frac{\left(A^{2}-1\right) \lambda^{2}}{\lambda^{2}-B}+\frac{C \lambda^{2}\left(\lambda^{2}-\lambda_{0}^{2}\right)}{\left(\lambda^{2}-\lambda_{0}^{2}\right)+\gamma^{2} \lambda^{2}}, \\
& \epsilon_{i}=\frac{C \lambda^{3} \gamma}{\left(\lambda^{2}-\lambda_{0}^{2}\right)+\gamma^{2} \lambda^{2}},
\end{aligned}
$$

where $\epsilon_{r}$ is the real part of the dielectric constant, $\epsilon_{i}$ is the imaginary part of the dielectric constant, $\lambda$ is the wavelength, $A, B$ are the Sellmeier coefficients, $\lambda_{0}$ is the central wavelength of the Lorentz absorption peak, $C$ is the amplitude of the Lorentz peak, and $\gamma$ is the width of the Lorenz peak.

Finally, a top layer (the rough surface in Fig. 3) was included to simulate the surface microroughness of the layer. Higher standard deviations result from regressions with simpler models. The regression was simultaneously performed over three measurements at different incidence angles $\left(54.93^{\circ}, 64.95^{\circ}\right.$, and $69.95^{\circ}$ ). Measuring at three angles increases the confidence about the parameters under adjustment, and it is absolutely necessary in order to extract accurate physical parameters from anisotropic samples and inhomogeneous films.

The values of the fit parameters obtained from the regression are shown in Table 1 . The refractive index versus $\lambda$ for sublayer 1 (the sublayer closest to the ambient) and for sublayer 20 (the sublayer closest to the substrate) can be observed in Fig. 4. Sublayer 20 has the highest value of the refractive index, and the difference between both sublayers is 0.234 at 633 $\mathrm{nm}(2.193$ at $633 \mathrm{~nm}$ and 2.327 at $633 \mathrm{~nm})$. This strong gradient is due to the low value of the substrate temperature during the deposition $\left(200^{\circ} \mathrm{C}, T_{s} /\right.$ $T_{m}=0.109$, zone 1 in the SZM). A higher degree of homogeneity layers can be obtained with the same deposition experimental setup when the substrate

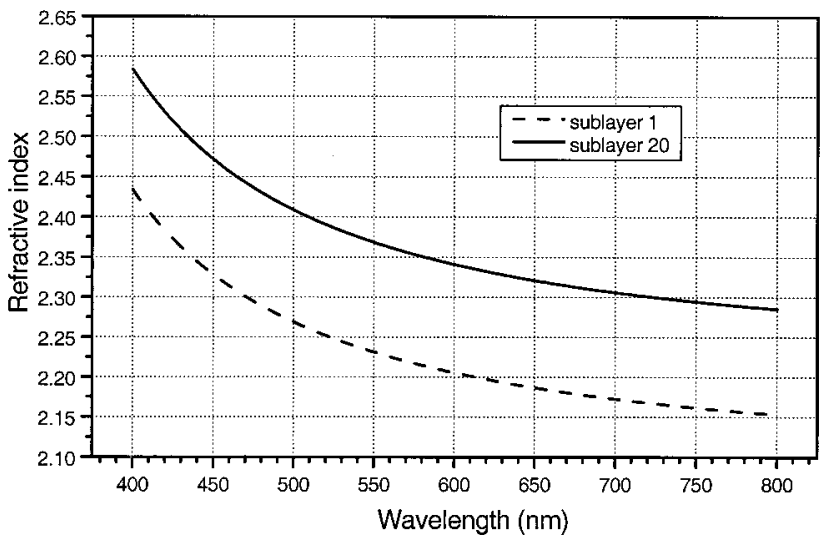

Fig. 4. Refractive indices of sublayer 1 (the closest one to the ambient) and sublayer 20 (the closest one to the substrate). The difference between both sublayers (inhomogeneity of the film) is more than 0.2. The ellipsometric measurements were carried out at $30 \% \pm 2 \% \mathrm{RH}$ and $23{ }^{\circ} \mathrm{C} \pm 1{ }^{\circ} \mathrm{C}$.

temperature is increased. The lower the substrate temperature, the higher the inhomogeneity of the film.

This fact is in complete agreement with the columnar structure of the film. The nanostructure of films deposited by evaporation methods presents a conical columnar shape. In the first stages of film deposition, ${ }^{25}$ small structural elements act as the basis for later-growing dentrites. Therefore the size of pores that are closest to the substrate is determined by the distance between these basic elements. During film-thickness growth, the structural units tend to cluster into larger groups. The voids in the group are closed, such that larger voids appear between the groups than between the dentrites, and these groups of dentrites form the observed columns. The agglomeration to larger columns, following that mechanism, proceeds with increasing film thickness. These larger voids form the greater pore size of the top surface of the film, and that explains the conical shape of the columns. By use of a low substrate temperature during the deposition, the conical structure (and therefore the inhomogeneity of the film) and, consequently, the porosity of the film are created. In this sense, porosity can be tailored, and this is useful for the design of specific sensors.

The marked gradient of the refractive index profiles other minor characteristics of the film such as anisotropy. A model that assumes anisotropy of the layer is due to the columnar structure of the film was tested. In this model the film is considered a uniaxial material with the optical axis normal to the surface of the film (following the columns' direction). The resulting regression has a higher, but acceptable, value $(0.0015)$ of the standard deviation. The ordinary refractive index obtained (polarization is normal to the optical axis; identical polarization to the film's TE guided mode) is 2.260 at $633 \mathrm{~nm}$, and the extraordinary refractive index is 2.273 at $633 \mathrm{~nm}$ (polarization is parallel to the optical axis; identical 


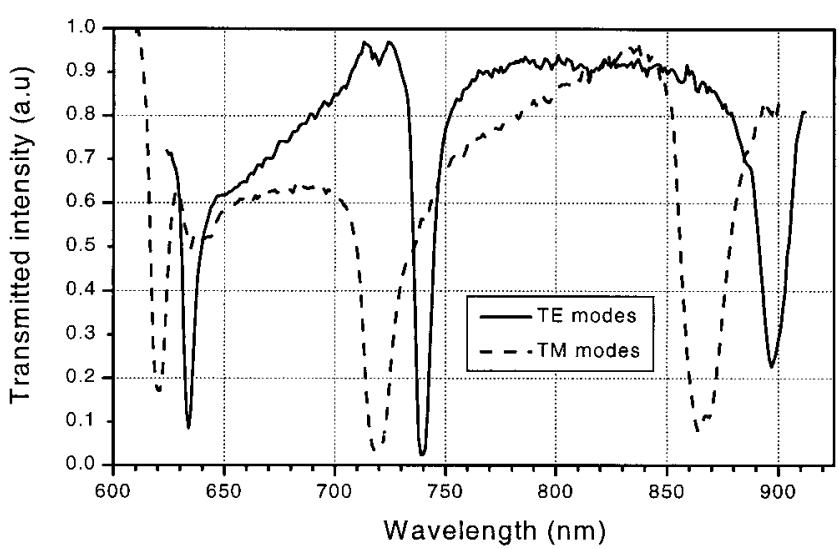

Fig. 5. Experimental spectral response of the SPF device with the $\mathrm{TiO}_{2}$ overlayer $\left(36 \% \pm 1 \% \mathrm{RH}, 21.5{ }^{\circ} \mathrm{C} \pm 0.5{ }^{\circ} \mathrm{C}\right)$. The coupling resonances with the TE and TM guided modes of the film are evidenced.

polarization to that of the film's TM guided mode). The difference obtained between the ordinary and the extraordinary refractive indices is 0.013 . These results are in agreement with Refs. 8 and 9. This value is 1 order of magnitude lower than the effect produced by the inhomogeneity. Therefore it cannot be stated that the film is anisotropic without other measurements that are complementary to the ellipsometric ones. It can be observed that the refractive indices for TE and TM polarizations are approximately equal to the averaged refractive index (2.26 at $633 \mathrm{~nm}$ ) obtained from the results of the inhomogeneity model. These results verify the coherence of our models.

\section{Side-Polished Fiber Resonances}

The experimental spectral response of the SPF device $\left(36 \% \pm 1 \% \mathrm{RH}\right.$ and $\left.21.5^{\circ} \mathrm{C} \pm 0.5{ }^{\circ} \mathrm{C}\right)$ is showed in Fig. 5. In this plot the resonances of the TE and TM coupling modes between the fiber and the film are displayed.

The theoretical model explained in Subsection 2.B and the values described in Subsection 3.A were used to simulate the experimental data. To simplify the model, we considered the film homogeneous. The optical dispersion of the components of the system (fiber and film) has also been taken into account. The dispersion law chosen for the fiber was a secondorder Sellmeier law ${ }^{26}$ :

$$
n(\lambda)=\left[1+\frac{\left(A^{2}-1\right) \lambda^{2}}{\lambda^{2}-B}\right]^{1 / 2} .
$$

The coefficients of the Sellmeier law for the effective index and the cladding refractive index of the fiber were

$$
\begin{aligned}
& A_{\text {effective }}=1.451, \\
& A_{\text {cladding }}=1.448, \\
& B_{\text {effective }}=B_{\text {cladding }}=0.009 .
\end{aligned}
$$

Taking all these considerations into account, we can fit the theoretical data to experimental points with a high degree of agreement if anisotropy is introduced. The film dispersion law and the total thickness of the film obtained from the ellipsometric measurements were used with a concentration of void equal to 0.05 for TM modes and 0.063 for TE modes. The different values of the void concentration for TE and TM provide the characterization of the film anisotropy. It corresponds to a value of the refractive index equal to 2.198 at $633 \mathrm{~nm}$ for TM polarization and 2.182 at $633 \mathrm{~nm}$ for TE polarization. We have assumed that the film is homogeneous to simplify the calculus of the resonances. Therefore we have obtained a pseudoaveraged value of the real refractiveindex profile. However, if the $\mathrm{TiO}_{2}$ layer measured by ellipsometry and the SPF overlayer were identical, a value of the refractive index around 2.26 would be expected. Therefore the pseudoaveraged value indicates the layers of both samples are slightly different in that the SPF overlayer has a higher void concentration (lower value of the refractive index). Nevertheless, when an anisotropic layer is considered, it can be observed that the difference between the refractive indices for the TM and TE polarizations is small: 0.013 by ellipsometry and 0.016 by SPF measurements. In addition, it is necessary to note that the dispersion law obtained by ellipsometry is directly introduced in the SPF theoretical model. The only modification was an increment of the void fraction. Hence the optical properties and the nanostructure of both samples are similar. Taking all these considerations into account, we can conclude that the results obtained with the theoretical models from the SPF data and from the ellipsometric ones are in complete agreement.

Figures 6(a) and 6(b) represent the perfect agreement between the experimental and the theoretical data and confirm the validity of the theoretical model and the ellipsometric measurements of the optical constants of the film. It can be concluded that the columnar shape of the nanostructure produces the anisotropy and the inhomogeneity of the coating. An absolute agreement between the nanostructure properties and optical properties of the film has been evidenced.

\section{B. Pore Size Distribution}

The behavior of sample $1\left(\mathrm{TiO}_{2}\right.$ over a glass substrate) under changes of $\mathrm{RH}$ was characterized by measurement of the changes in the ellipsometric parameter cos $\Delta$ at a wavelength of $633 \mathrm{~nm}$. Sample 2 $\left(\mathrm{TiO}_{2}\right.$ over the SPF) was studied by measurement of the resonance shifts at the same wavelength. These water adsorption isotherms provide information about the shape and the size of the pores.

Figure 7 shows the adsorption isotherm for sample $1\left(T=25.5^{\circ} \mathrm{C} \pm 0.5^{\circ} \mathrm{C}\right)$, and Fig. 8 shows the behavior of sample $2\left(T=26.1{ }^{\circ} \mathrm{C} \pm 0.6{ }^{\circ} \mathrm{C}\right)$. Both plots present a lack of hysteresis for the adsorption and desorption branches. The small differences between data of both branches are due to experimental uncer- 


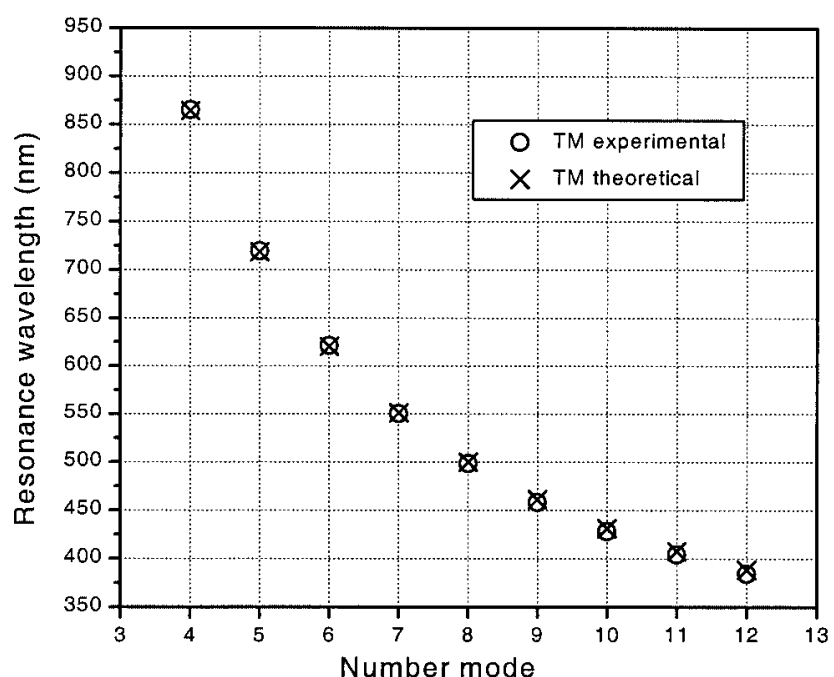

(a)

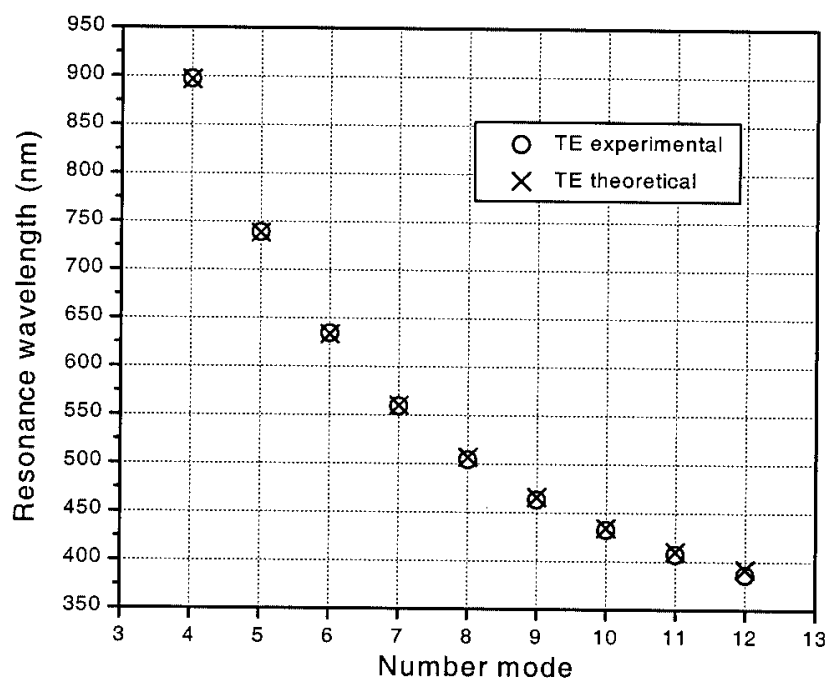

(b)

Fig. 6. Experimental and theoretical data of the resonances of the (a) $\mathrm{TM}$ and (b) $\mathrm{TE}$ modes of the $\mathrm{SPF}-\mathrm{TiO}_{2}$ system. Experimental data measured at $36 \% \pm 1 \% \mathrm{RH}$ and $21.5^{\circ} \mathrm{C} \pm 0.5{ }^{\circ} \mathrm{C}$.

tainties in the measurement. It is known ${ }^{18}$ that cone-shaped pores give a hysteresis-free behavior, and this shape is in complete agreement with the expected nanostructure of evaporated films.

Figure 9 displays the resulting pore size distributions calculated from these experimental isotherms with the Pierce method. The pore size distributions have been normalized. The results are summarized in Table 2. The most probable radius is defined as the radius value corresponding to the maximum of the pore size distribution, and the average radius is

$$
r_{\text {average }}=\frac{\int_{0}^{\infty} r_{p} \frac{\mathrm{d} V}{\mathrm{~d} r_{p}} \mathrm{~d} r_{p}}{\int_{0}^{\infty} \frac{\mathrm{d} V}{\mathrm{~d} r_{p}} \mathrm{~d} r_{p}} .
$$

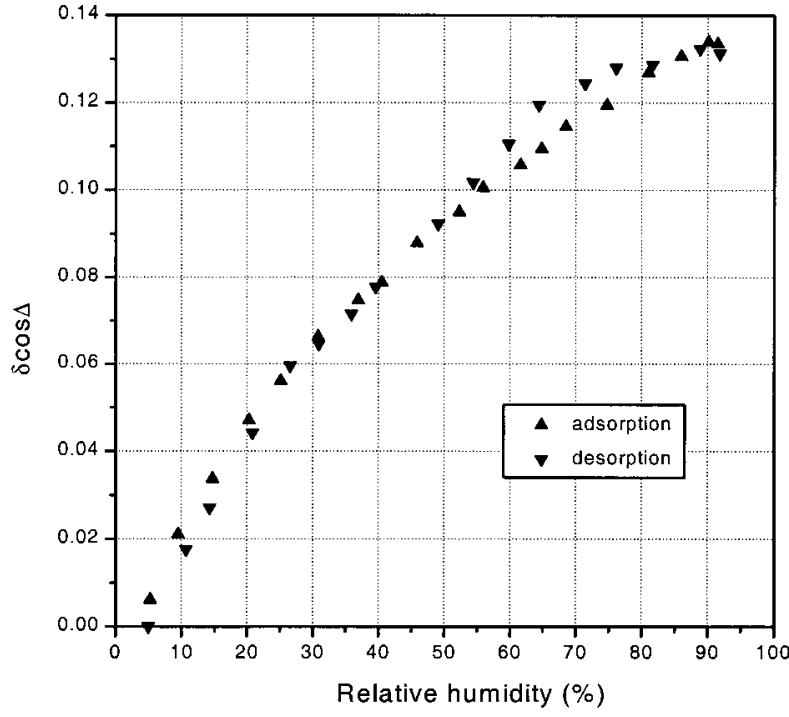

Fig. 7. Water adsorption isotherm for sample 1 by ellipsometric measurements $\left(T=25.5^{\circ} \mathrm{C} \pm 0.5^{\circ} \mathrm{C}\right)$.

The obtained values are in the range from $\sim 8.5$ to $\sim 25 \AA$. This is the limit of the validity of the Kelvin equation. The Kelvin equation, and therefore the Pierce method, could be not applicable to microporous materials (less than $20 \AA$ ) whose pore widths are a few molecular diameters of adsorbate. For nitrogen, the limit of the pore radius is estimated as approximately $17 \AA$, which corresponds approximately to four molecules (the nitrogen molecule's diameter is $\sim 3.7 \AA$ ). In our study we used water as adsorbate. The water molecular diameter is approximately $\sim 1.3$ $\AA$, although it depends on the molecular position. The nitrogen molecule is three times bigger, so we assumed that the $\sim 17 \AA$ limit can be reduced. The pore radii of the titania film under study are $\sim 10 \AA$, corresponding to approximately eight molecules of

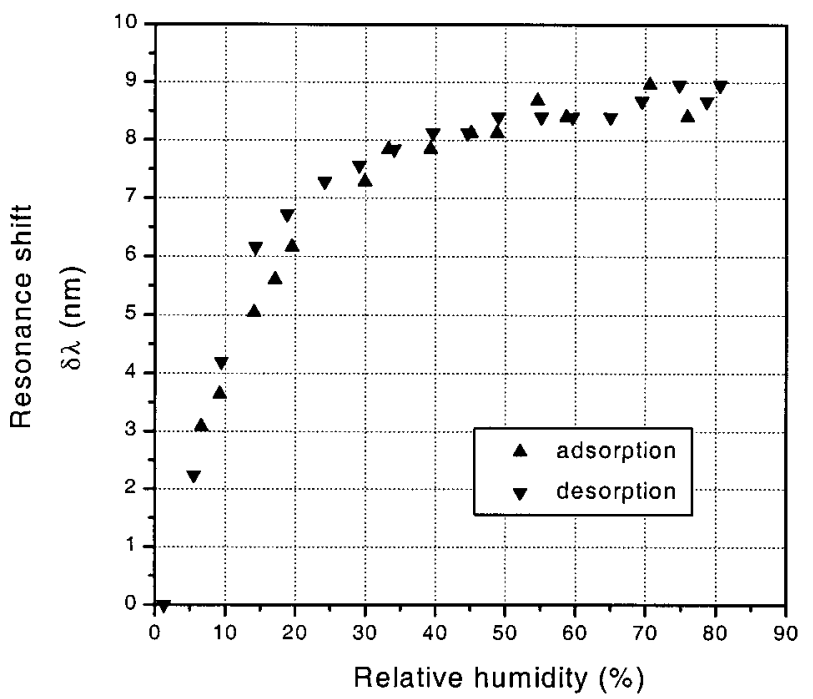

Fig. 8. Water adsorption isotherm for sample 2 by resonance shift of SPF measurements $\left(T=26.1{ }^{\circ} \mathrm{C} \pm 0.6{ }^{\circ} \mathrm{C}\right)$. 


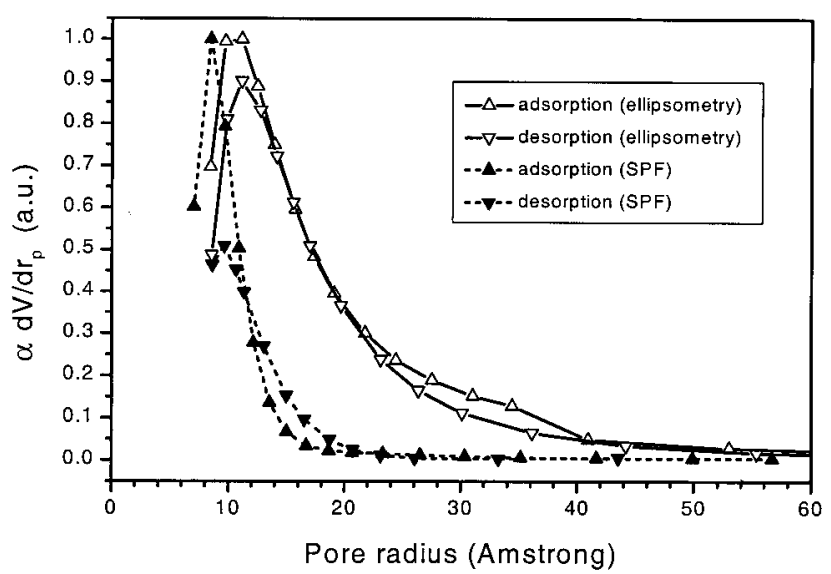

Fig. 9. Pore size distribution calculated with the Pierce method from the ellipsometric and the resonance shift data (Figs. 7 and 8, respectively).

water. Therefore we are close to the validity limit of the Kelvin equation, but it can be still used as a rough method. Of course, the tensile strength and the Kelvin equation are modified, and, probably, the real value of the radius will be higher as is explained in Ref. 18.

It can be observed that the values of the average radius and the most probable radius obtained from the ellipsometric measurements are higher than the SPF probe. As was explained in Subsection 2.B, the SPF device has a high sensitivity to the area of the filmfiber interface, and the ellipsometry provides a global characterization for the transparent layer. Hence it can be concluded that the pore radii of the filmsubstrate interface are lower than those of the filmambient interface. Again, this agrees with the assumption that columns have a conical shape.

The different sensitivity of both techniques also explains the difference in values between the most probable radius and average radius obtained by ellipsometry and those values from the SPF device measurements. Note that the pore size distribution obtained by ellipsometry is wider than the distribution obtained from the SPF device because all the pores along the thickness of the transparent layer contribute to the adsorption measurement. In the case of the SPF device, only the pores close to the film-fiber interface contribute, and therefore the size pore distribution is narrower. For a wide distribution, the most probable value can be different from the average value.

In conclusion, we are able to determine the inhomogeneity produced by the dependence of the poros-

Table 2. Results of Size Pore Distribution Obtained from the Ellipsometric Data and the Resonance Shift of the SPF Device

\begin{tabular}{lccccc}
\hline & \multicolumn{2}{c}{ Average Radius } & & \multicolumn{2}{c}{ Most Probable Radius } \\
\cline { 2 - 3 } \cline { 5 - 6 } Pore Size $(\AA)$ & Adsorption & Desorption & & Adsorption & Desorption \\
\hline Ellipsometry & 24.9 & 23.4 & & 11.1 & 11.4 \\
SPF & 12.1 & 12.9 & & 8.5 & 9.6 \\
\hline
\end{tabular}

ity on thickness of the film by using a combination of both techniques. The fact of different sensitivities of the ellipsometric measurements and the resonance shifts of the SPF permits us to characterize the film properties along its thickness.

\section{Correlation Between Nanostructure and Optical Properties of the Film}

Summarizing the previous results, we can establish a relationship between the optical properties of these films and their porous nanostructure:

1. Anisotropy of the film has been detected by a SPF device, and it has been verified by ellipsometry. The film is uniaxial with the optical axis normal to the surface. It is in agreement with a columnar structure crossing both film sides.

2 . Inhomogeneity has been characterized by ellipsometry and corresponds to a greater material density (higher refractive index) in the film-substrate interface than the density in the film-ambient surface (lower refractive index). This is due to the conical shape of the columns formed during the growth process.

3. Optical properties' changes with humidity have been studied. Magnitudes linked to the refractive index of the layer have been studied under different humidity conditions by ellipsometric and SPF probe techniques. The lack of hysteresis in the adsorption isotherms is in agreement with the conical columns of the nanostructure.

4. Finally, the pore size distribution obtained from the adsorption isotherm data confirms the smaller pore sizes in the substrate-film interface, which agrees with the conical-shaped columns. It is necessary to emphasize the novel, to our knowledge, application of the Pierce method to SPF devices in order to obtain the pore size distribution from the resonances shifts of the SPF device.

\section{Conclusion}

The application of the Pierce method to the shift of the resonance of a SPF device during an adsorptiondesorption cycle provides information about the pore size distribution of porous films.

Correlation between measurements performed with a SPF and spectroscopic ellipsometry has been demonstrated. A characterization of the film porosity can be achieved with a combination of both techniques. As film porosity can be tailored, studies can be carried on to optimize the water adsorptiondesorption in specific material for thin-film coatings. Traditional methods of adsorption isotherms are volumetric and gravimetric. Thin films are not suitable to be tested with such methods because the adsorptive medium is quite reduced. The correlation between nanostructure and optical properties has become better known from our measurements. The influence on optical parameters in devices that use thin-film coatings can be predicted with these techniques. 
The authors thank A. J. Fort for his helpful work on the growth of the $\mathrm{TiO}_{2}$ films.

\section{References}

1. J. M. Bennett, E. Pelletier, G. Albrand, J. P. Borgogno, B. Lazarides, C. K. Carniglia, R. A. Schmell, T. H. Allen, T. Tuttle-Hart, K. H. Guenther, and A. Saxer, "Comparison of the properties of titanium dioxide films prepared by various techniques," Appl. Opt. 28, 3303-3317 (1989).

2. J. S. Chen, S. Chao, J. S. Kao, G. R. Lai, and W. H. Wang, "Substrate-dependent optical absorption characteristics of titanium dioxide thin films," Appl. Opt. 36, 4403-4408 (1997).

3. B. A. Movchan and A. V. Demchishin, "Study of the structure and properties of thick vacuum condensates of nickel, titanium, tungsten, aluminium oxide and zirconium dioxide," Phys. Met. Metallogr. (USSR) 28, 83-90 (1969).

4. J. A. Thornton, "High rate thick film growth," Annu. Rev. Mater. Sci. 7, 239-260 (1977).

5. R. Messier, A. P. Giri, and R. A. Roy, "Revised structure zone model for thin film physical structure," J. Vac. Sci. Technol. A 2, 500-503 (1984).

6. A. G. Dirks and H. J. Leamy, "Columnar nanostructure in vapor deposited thin films," Thin Solid Films 45, 219-323 (1977).

7. P. Ramanlal and L. M. Sander, "Theory of ballistic aggregation," Phys. Rev. Lett. 54, 1828-1831 (1985).

8. I. Hodgkinson, Q. H. Wu, and J. Hazel, "Empirical equations for the principal refractive indices and column angle of obliquely deposited films of tantalum oxide, titanium oxide, and zirconium oxide," Appl. Opt. 37, 2653-2659 (1998).

9. F. Flory, D. Endelema, E. Pelletier, and I. Hodgkinson, "Anisotropy in thin films: modeling and measurement of guided and nonguided optical properties: application to $\mathrm{TiO}_{2}$ films," Appl. Opt. 32, 5649-5659 (1993).

10. I. Hodgkinson, J. Hazel, and Q. H. Wu, "In situ measurement of principal refractive indices of thin films by two-angle ellipsometry," Thin Solid Films 313-314, 368-372 (1998).

11. C. K. Carniglia, "Ellipsometric calculation for nonabsorbing thin films with linear refractive-index gradients," J. Opt. Soc. Am. A 7, 848-856 (1990).

12. Md. Mosaddeq-ur-Rahman, G. Yu, K. M. Krishna, T. Soga, J.
Watanabe, T. Jimbo, and M. Umeno, "Determination of optical constants of solgel-derived inhomogeneous $\mathrm{TiO} 2$ films by spectroscopic ellipsometry and transmission spectroscopy," Appl. Opt. 37, 691-697 (1998).

13. G. Parjadis de Lariviére, J. M. Frigerio, F. Bridou, and J. Rivory, "Modelling of ellipsometric data of inhomogeneous TiO2 films," Thin Solid Films 233-234, 458-462 (1993).

14. S. Y. Kim, "Simultaneous determination of refractive index, extinction coefficient, and void distribution of titanium dioxide thin film by optical methods," Appl. Opt. 35, 6703-6707 (1996).

15. A. Alvarez-Herrero, A. J. Fort, H. Guerrero, and E. Bernabeu, "Ellipsometric characterization and influence of relative humidity on TiO2 layers optical properties," Thin Solid Films 349, 212-219 (1999).

16. A. Álvarez-Herrero, R. L. Heredero, E. Bernabeu, and D. Levy, "Adsorption of water on porous Vycor glass studied by ellipsometry," Appl. Opt. 40, 527-532 (2001).

17. B. P. Pal and G. R. Chakravarty, "All-fiber wavelength selective components for optical communication," Commun. Instr. 5, 181-208 (1997).

18. S. J. Gregg and K. S. W. Sing, Adsorption, Surface Area and Porosity (Academic, New York, 1997).

19. R. M. A. Azzam and N. N. Bashara, Ellipsometry and Polarized Light (North Holland, Amsterdam, 1977).

20. R. W. Collins, D. E. Aspnes, and E. A. Irene, "Proceedings of the Second International Conference on Spectroscopic Ellipsometry," Thin Solid Films 313-314 (1998).

21. V. A. Tolmachev, "Adsorption-ellipsometry method of studying the optical profile, thickness, and porosity of thin films," J. Opt. Technol. 66, 596-607 (1999).

22. V. A. Tolmachev, "Determination of the porosity of uniform films by adsorption-ellipsometric method," Opt. Spectrosc. 84, 584-588 (1998).

23. P. K. Tien, "Light waves in thin films and integrated optics," Appl. Opt. 10, 2395-2413 (1971).

24. D. E. Aspnes and A. A. Studna, "High precision scanning ellipsometer," Appl. Opt. 14, 220-228 (1975).

25. H. K. Pulker, Coatings on Glass (Elsevier, Amsterdam, 1999).

26. M. Born and E. Wolf, Principles of Optics (Pergamon, Oxford, UK, 1993). 